\title{
Single Cobalt Sites in Mesoporous N-Doped Carbon Matrix for Selective Catalytic Hydrogenation of Nitroarenes
}

Xiaohui Sun ${ }^{1}$, Alma I. Olivos-Suarez ${ }^{1}$, Dmitrii Osadchii ${ }^{1}$, Maria Jose Valero Romero ${ }^{1}$, Freek Kapteijn ${ }^{1}$, and Jorge Gascon ${ }^{2,1}$ *

${ }^{1}$ Catalysis Engineering, Department of Chemical Engineering, Delft University of Technology, Van der Maasweg 9, 2629 HZ Delft, The Netherlands

${ }^{2}$ King Abdullah University of Science and Technology, KAUST Catalysis Center, Advanced Catalytic Materials, Thuwal 23955, Saudi Arabia

${ }^{*}$ Correspondence to: jorge.gascon@kaust.edu.sa

Abstract: A supported cobalt catalyst with atomically dispersed Co- $\mathrm{N}_{\mathrm{x}}$ sites (3.5 wt.\% Co) in a mesoporous N-doped carbon matrix (named Co@mesoNC) is synthesized by hydrolysis of tetramethyl orthosilicate (TMOS) in a $\mathrm{Zn} / \mathrm{Co}$ bimetallic zeolitic imidazolate framework (BIMZIF(Co,Zn)), followed by high-temperature pyrolysis and $\mathrm{SiO}_{2}$ leaching. A combination of TEM, XRD XPS and X-ray absorption spectroscopy studies confirm the absence of cobalt nanoparticles and indicate that these highly dispersed cobalt species are present in the form of Co- $\mathrm{N}_{\mathrm{x}}$. The exclusive formation of Co- $\mathrm{N}_{\mathrm{x}}$ sites in the carbon matrix is attributed to the presence of a large amount of $\mathrm{Zn}$ and $\mathrm{N}$ in the BIMZIF precursor together with the presence of $\mathrm{SiO}_{2}$ in the pore space of this framework, extending the initial spatial distance between cobalt atoms and thereby impeding their agglomeration. The presence of $\mathrm{SiO}_{2}$ during hightemperature pyrolysis is proven crucial to create mesoporosity and a high BET area and pore volume in the $\mathrm{N}$-doped carbon support $\left(1780 \mathrm{~m}^{2} \mathrm{~g}^{-1}, 1.54 \mathrm{~cm}^{3} \mathrm{~g}^{-1}\right)$. This heterogeneous Co@mesoNC catalyst displays high activity and selectivity (>99\%) for the selective hydrogenation of nitrobenzene to aniline at mild conditions (0.5-3 MPa, 343-383 K). When more challenging substrates (functionalized nitroarenes) are hydrogenated, the catalyst Co@mesoNC displays an excellent chemoselectivity to the corresponding substituted anilines. The presence of mesoporosity improves mass transport of reactants and/or products and the accessibility of the active $\mathrm{Co}-\mathrm{N}_{\mathrm{x}}$ sites, and greatly reduces deactivation due to fouling.

Keywords: nitroarene, hydrogenation, Co single atom, chemoselectivity. 


\section{INTRODUCTION}

The reduction of nitroarenes to their substituted anilines is of great importance in organic synthesis and applied on large scale for the production of dyes, pharmaceuticals, pigments, and agrochemicals [1-4]. Hydrogen is attractive because it is one of the cleanest reductant [57]. Despite the development of a large number of molecular catalysts [8-12], in general these homogeneous catalysts suffer from difficulties in recycling and separation from products. Hence, heterogeneous catalysts containing supported metal nanoparticles (NPs) are more attractive [13-18]. Platinum-group metals have been proposed as alternative and are industrially used in the direct hydrogenation of nitroarenes [19-23]. Although they are highly efficient in the activation of nitro groups, these catalysts are also highly active for hydrogenolysis of carbon-halogen bonds (i.e. $-\mathrm{F},-\mathrm{Cl}$ etc.) and hydrogenation of other reducible groups (i.e. $\mathrm{C}=\mathrm{O}$ and $\mathrm{C}=\mathrm{C}$ etc.), which usually leads to poor chemoselectivity [2428]. Thus, the exploration for advanced materials that catalyze the hydrogenation of nitroarenes maintaining high activities and without compromising chemoselectivity remains challenging.

Recently, tremendous efforts have led to the development of cheaper first-row transitionmetal-based hydrogenation catalysts (i.e. Fe, Co, and Ni) [15, 29-41]. Among these catalysts, $\mathrm{N}$-doped carbon supported cobalt materials prepared by the carbonization of metal-organicframeworks (MOFs) or a mixture of cobalt salts and organic complex exhibited good activity and chemoselectivity in the hydrogenation of nitroarenes [29-32, 36, 39-46]. There is a general agreement that cobalt plays an indispensable role in enhancing the hydrogenation activity of the catalysts. Yet, the heterogeneity of the cobalt species (i.e. accessible and inaccessible cobalt nanoparticles encapsulated by graphite shells, single cobalt atoms coordinated with nitrogen $\left(\mathrm{Co}-\mathrm{N}_{\mathrm{x}}\right)$ etc. $)$ raises debate on what is the real active site in the hydrogenation process, and therefore greatly hinders the rational design and development of highly active and durable catalysts. Very recently, by one-step pyrolysis of a cobalt-containing MOF (ZIF-67) under $\mathrm{N}_{2}$ atmosphere followed by a subsequent acid treatment, we proved that the accessible cobalt nanoparticles in that system exhibit a high activity for this reaction [36]. Surprisingly, after acid leaching, the sample still shows outstanding hydrogenation activity. Although highly dispersed cobalt species (i.e. Co- $\mathrm{N}_{\mathrm{x}}$ ) are considered to exist in the acidleached sample, the presence of those acid-resistant cobalt nanoparticles, fully encapsulated by multilayer graphitic carbon shells largely impedes further exploration of the structureactivity relation in this reaction. Hence, a rational design strategy that can preferentially produce active $\mathrm{Co}-\mathrm{N}_{\mathrm{x}}$ sites should be explored. 
In view of the similar coordination of $\mathrm{Co}^{2+}$ and $\mathrm{Zn}^{2+}$ with 2-methylimidazole in ZIF-67 and ZIF-8 [47-49], a series of $\mathrm{Zn} / \mathrm{Co}$ bimetallic zeolitic imidazolate framework (BIMZIF $(\mathrm{Co}, \mathrm{Zn}))$ have been recently reported, where the spatial distribution of Co and $\mathrm{Zn}$ in the framework is largely dependent on sequential addition of the metal precursors during synthesis [50-54]. Remarkably, when the precursors are added together, a homogeneous distribution of $\mathrm{Zn}$ and $\mathrm{Co}$ in the $\operatorname{BIMZIF}(\mathrm{Co}, \mathrm{Zn})$ can be obtained. The presence of $\mathrm{Zn}^{2+}$ effectively separates Co atoms in the framework, and, to some extent, precludes their agglomeration into cobalt NPs during the high-temperature pyrolysis [52, 53]. The carbon matrix generated using this strategy exhibits high surface area but normally microporosity [52, 53], which can lead to significant internal mass transport limitations during the catalytic process, and therefore catalytic activity and stability can be severely inhibited $[55,56]$.

Herein, we report a facile and easily scalable method to construct an atomically dispersed cobalt catalyst with a 3.5 wt.\% Co loading in a mesoporous N-doped carbon for chemoselective hydrogenation of nitroarenes to their substituted anilines under mild conditions. The catalyst is synthesized in several steps: (i) hydrolysis of tetramethyl orthosilicate (TMOS) in the pores of a BIMZIF(Co,Zn), followed by (ii) high-temperature pyrolysis and (iii) silica leaching. We demonstrate that the high $\mathrm{Zn} / \mathrm{Co}$ molar ratio in the parent BIMZIF together with the $\mathrm{SiO}_{2}$-protection strategy is crucial to impede the formation of cobalt nanoparticles and preferentially generate atomically dispersed $\mathrm{Co}-\mathrm{N}_{\mathrm{x}}$ sites. At the same time, the $\mathrm{SiO}_{2}$-templated approach maintains a high specific surface area and generates mesoporosity in the N-doped carbon matrix. These Co- $\mathrm{N}_{\mathrm{x}}$ sites in the mesoporous $\mathrm{N}$-doped carbon matrix exhibit superior hydrogenation activity and chemoselectivity, and good catalytic stability. This strategy opens a path for the rational designing of highly active nonnoble catalysts for hydrogenation reactions.

\section{EXPERIMENTAL}

\subsection{Materials}

2-Methylimidazole (MeIm, purity 99\%), zinc nitrate hexahydrate $\left(\mathrm{Zn}\left(\mathrm{NO}_{3}\right)_{2} \cdot 6 \mathrm{H}_{2} \mathrm{O},>98 \%\right)$, cobalt nitrate hexahydrate $\left(\mathrm{Co}\left(\mathrm{NO}_{3}\right)_{2} \cdot 6 \mathrm{H}_{2} \mathrm{O},>99 \%\right)$, tetramethyl orthosilicate (TMOS $\geq 99 \%$ ), and methanol (>99.8\%) were purchased from Sigma-Aldrich Chemical Co. All the chemicals were used without further purification.

\subsection{Catalyst synthesis}

For the synthesis of BIMZIF(Co, $\mathrm{Zn})$, a mixture of $\mathrm{Zn}\left(\mathrm{NO}_{3}\right)_{2} \cdot 6 \mathrm{H}_{2} \mathrm{O}$ and $\mathrm{Co}\left(\mathrm{NO}_{3}\right)_{2} \cdot 6 \mathrm{H}_{2} \mathrm{O}$ with $\mathrm{Zn}^{2+} / \mathrm{Co}^{2+}$ molar ratio of 25 was dissolved in $200 \mathrm{~mL}$ methanol. A mixture of MeIm 
$(6.489 \mathrm{~g})$ in $200 \mathrm{~mL}$ methanol was rapidly poured into the above solution with vigorous stirring for $24 \mathrm{~h}$ at room temperature [52]. The total molar amount of $\left(\mathrm{Co}^{2+}+\mathrm{Zn}^{2+}\right)$ was fixed to be $10 \mathrm{mmol}$. Afterwards, the products were collected by filtration, washed thoroughly with methanol, and dried overnight at $353 \mathrm{~K}$ under vacuum. For the synthesis of ZIF-8(Zn) and ZIF-67(Co), all the steps were the same except only $\mathrm{Zn}\left(\mathrm{NO}_{3}\right)_{2} \cdot 6 \mathrm{H}_{2} \mathrm{O}$ or $\mathrm{Co}\left(\mathrm{NO}_{3}\right)_{2} \cdot 6 \mathrm{H}_{2} \mathrm{O}$ and MeIm were used. Around $1 \mathrm{~g}$ ZIF materials can be produced from each batch.

$1 \mathrm{~g}$ of the synthesized BIMZIF(Co,Zn) was suspended in $5 \mathrm{~mL}$ TMOS in an autoclave, and further transferred into a rotation oven and heated up to $333 \mathrm{~K}$ overnight. After the oven was cooled down to room temperature, the mixture was carefully washed with $0.5 \mathrm{~mL}$ ethanol by filtration. Then the obtained BIMZIF@TMOS material was placed in a cotton thimble of $22 \mathrm{~mm}$ diameter inside a glass tube of $25 \mathrm{~mm}$ diameter. The glass tube was fitted to a round bottom flask containing $500 \mathrm{ml}$ of water. A needle to bubble $10 \mathrm{ml} \mathrm{min}^{-1}$ of $\mathrm{N}_{2}$ flux into water was also fitted. The temperature was raised to $323 \mathrm{~K}$ to create a wet $\mathrm{N}_{2}$ stream to hydrolyze the TMOS molecules for $24 \mathrm{~h}$, after which the sample was collected and dried in an oven at $333 \mathrm{~K}$ overnight. The obtained sample was denoted as BIMZIF@ $\mathrm{SiO}_{2}$, with the yield of $1.2 \mathrm{~g}$.

$\mathrm{Co} @ \mathrm{NC}-\mathrm{SiO}_{2}$ was prepared by pyrolysis of 1 g BIMZIF@ $\mathrm{SiO}_{2}$ at $1173 \mathrm{~K}$ for $4 \mathrm{~h}$ under $\mathrm{N}_{2}$ at a ramp of $2 \mathrm{~K} \mathrm{~min}^{-1}$, with the yield of $0.44 \mathrm{~g}$. The obtained $\mathrm{Co} @ \mathrm{NC}-\mathrm{SiO}_{2}$ was further leached in $1 \mathrm{M} \mathrm{NaOH}$ solution for $24 \mathrm{~h}$ to remove the $\mathrm{SiO}_{2}$ template, followed by washing with deionized water until the $\mathrm{pH}$ reached neutral, and dried at $323 \mathrm{~K}$ overnight under vacuum to afford Co@mesoNC sample, with the yield of $0.23 \mathrm{~g}$.

For comparison, Co@NC and NC were prepared by pyrolysis of $1 \mathrm{~g} \operatorname{BIMZIF}(\mathrm{Co}, \mathrm{Zn})$ and ZIF-8 at $1173 \mathrm{~K}$ for $4 \mathrm{~h}$ under $\mathrm{N}_{2}$ at a ramp of $2 \mathrm{~K} \mathrm{~min}^{-1}$, respectively. The pyrolyzed ZIF-67 sample was obtained by pyrolysis of ZIF-67 at $1073 \mathrm{~K}$ for $8 \mathrm{~h}$ under $\mathrm{N}_{2}$ using a temperature ramp of $2 \mathrm{~K} \mathrm{~min}^{-1}$.

\subsection{Characterization}

X-ray diffraction (XRD) patterns were recorded on a Bruker D8 Advance X-ray diffractometer equipped with a Co-Ka radiation $(\lambda=0.179026 \mathrm{~nm})$. Transmission electron microscopy (TEM) and high-resolution TEM (HR-TEM) were performed by using a Talos F200X microscope (FEI, Hillsboro, OR, USA) at an acceleration voltage of $200 \mathrm{kV}$. Raman spectra were obtained with a commercial Renishaw in Via Reflex confocal microscope using a $532 \mathrm{~nm}$ laser. Measurements were carried out in samples without any pre-treatment at ambient conditions. The $\mathrm{N}_{2}$ adsorption-desorption measurements were performed by using a Micromeritics Tristar 3020 apparatus at $77 \mathrm{~K}$ [57]. Prior to measurement, samples were degassed under vacuum at $383 \mathrm{~K}$ overnight. The Co and $\mathrm{N}$ contents in the samples were 
measured by atomic adsorption spectroscopy (AAS) (Analyst 200, Perkin Elmer, USA) and elemental analysis (Vario EL, Elementar, Germany), respectively. XPS measurements were performed on a $K$-alpha Thermo Fisher Scientific spectrometer using monochromatic Al- $K \alpha$ radiation at ambient temperature and chamber pressure of about $10^{-8} \mathrm{mbar}$. All the spectra measured were corrected by setting the reference binding energy of carbon $(1 s)$ at $284.8 \mathrm{eV}$.

X-ray absorption experiments were performed at DUBBLE beam line BM26A in ESRF, Grenoble. The materials were studied using Co K-edge. Energy calibration was achieved using metal foil as reference and the first peak in the first derivative of the metal foil XAS spectrum was calibrated to $7709 \mathrm{eV}$ [58]. Calibration and data alignment was performed using Athena and reference compounds values were obtained from Hephaestus. After averaging, the spectra of the studied samples were normalized to a total absorption of unity and processed using the Athena data normalization and analysis package [59]. For normalization, the spline $r$-background parameter was set to 1.0. The background subtraction was carried using a preedge range of -200 to $-30 \mathrm{eV}$ and a post-edge linear range of 50 to $985 \mathrm{eV}$. A spline range of $k$ $=0$ to $k=14.8 \mathrm{~A}^{-1}$ and $k$-weight of 3 was used to isolate the EXAFS (chi)function. The Co $K$ edge EXAFS spectra were Fourier transformed over a $k$-range of $k=3$ to $k=11 \AA^{-1}$. Fitting of Co K-edge EXAFS spectra was carried out by Artemis software.[60] The spectra of the different samples were fitted in $R$-space with a $R$ range of 1 to $4.5 \AA$, and the fittings were done with a $k$-weight of 3 . The Fourier transform and inverse Fourier transform was carried with a $\Delta \mathrm{k}$ of 1 and $\Delta \mathrm{R}$ of 0 . From the references fits we obtain the amplitude factor $\left(\mathrm{S}_{0}{ }^{2}\right)$ equal to $0.77 \pm 0.03$ for cobalt foil and to $0.73 \pm 0.13$ for $\mathrm{CoO}$. In further fittings of the experimental samples $S_{0}^{2}=0.73$ was used.

\subsection{Catalyst performance}

In a typical experiment, hydrogenation reactions are carried out in a batch mode, for which the autoclave is filled with nitrobenzene $(1 \mathrm{mmol})$, an internal standard (dodecane, 0.65 mmol), the Co@mesoNC catalyst with a substrate to cobalt molar ratio of 150, and ethanol (5 $\mathrm{ml}$ ) as solvent. Before starting the reaction, the autoclaves are purged 3 times with $\mathrm{He}$ to remove air, and pressurized to $3 \mathrm{MPa} \mathrm{H}_{2}$, followed by heating to $383 \mathrm{~K}$. After a fixed reaction time, the autoclaves are cooled down to room temperature and the hydrogen pressure is carefully released. The stirring speed is kept at $800 \mathrm{rpm}$ to avoid mass transfer limitations. For recycling studies, the reaction was performed under the same conditions as mentioned above except using the recovered catalyst. The used catalyst was filtered and washed thoroughly with ethanol, dried under vacuum at $313 \mathrm{~K}$ overnight and then used for the next run without 
any reactivation or purification. Conversions of all substrates were determined by gas chromatography (GC) analysis.

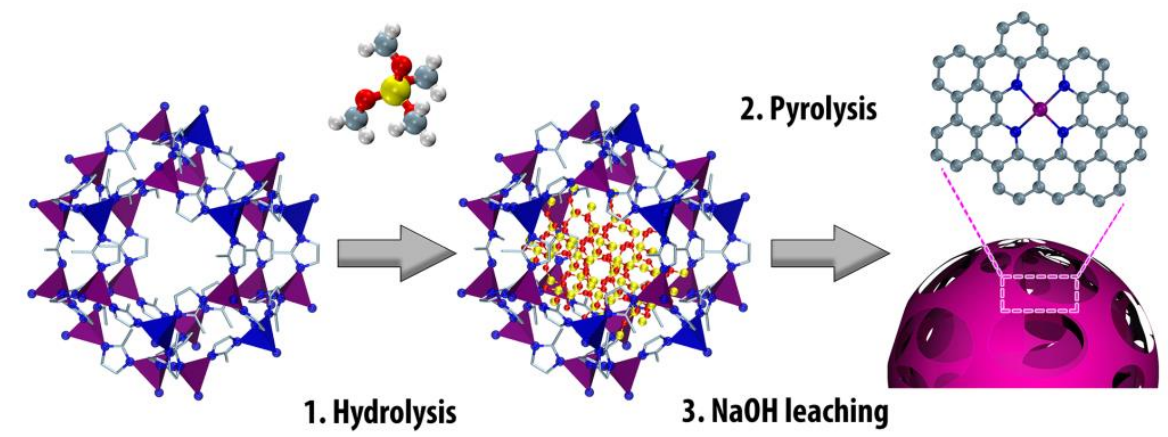

Figure 1: Schematic illustration of the synthesis of the Co@mesoNC catalyst. (1) Impregnation and hydrolysis of TMOS molecules in the porosity of BIMZIF. (2) Pyrolysis of the mixture of

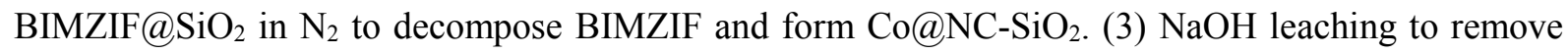
$\mathrm{SiO}_{2}$ to generate the $\mathrm{Co} @$ mesoNC catalyst.

\section{RESULTS AND DISCUSSION}

\subsection{Preparation and Characterization of the Co@mesoNC catalyst}

Figure 1 illustrates the preparation process for the Co@mesoNC catalyst. In the first step, the BIMZIF(Co,Zn) was synthesized by reacting $\mathrm{Co}^{2+}$ and $\mathrm{Zn}^{2+}$ ions with 2-methylimidazole (MeIm) in methanol. Then tetramethyl orthosilicate (TMOS) was introduced into the pores of BIMZIF followed by hydrolysis to obtain BIMZIF@ $\mathrm{SiO}_{2}$ material. Finally, the BIMZIF@SiO $\mathrm{Sim}_{2}$ sample was pyrolyzed at $1173 \mathrm{~K}$, and the $\mathrm{SiO}_{2}$ template was subsequently leached with a $\mathrm{NaOH}$ solution to generate the $\mathrm{Co} @$ mesoNC catalyst (see Experiment Section for the details).

The powder X-ray diffraction (XRD) pattern (Fig. S1) of the bimetallic ZIF matches well with ZIF-8 (Zn), confirming the successful synthesis [36, 48]. High-angle annular dark-field scanning transmission electron microscopy (HAADF-STEM) (Fig. S2a) and energy-dispersive X-ray spectroscopy (EDX) (Fig. S2b-e) images of the prepared BIMZIF crystals with a size of 30-50 nm clearly exhibit a typical rhombic dodecahedral shape with homogeneously dispersed cobalt, zinc, carbon and nitrogen throughout the crystals. The tracking of each synthetic step for Co@mesoNC by TEM combined with elemental mapping analysis confirms the uniform distribution of Si and Co in both BIMZIF@SiO 2 (Fig. S3a-c) and $\mathrm{Co} @ \mathrm{NC}-\mathrm{SiO}_{2}$ materials (Fig. S3d-f), and highlights the absence of cobalt nanoparticles in 
$\mathrm{Co} @ \mathrm{NC}^{-\mathrm{SiO}_{2}}$ (Fig. S4). After removal of the $\mathrm{SiO}_{2}$ template, the absence of cobalt nanoparticles in the Co@mesoNC catalyst is further confirmed in Figure 2a,b, while

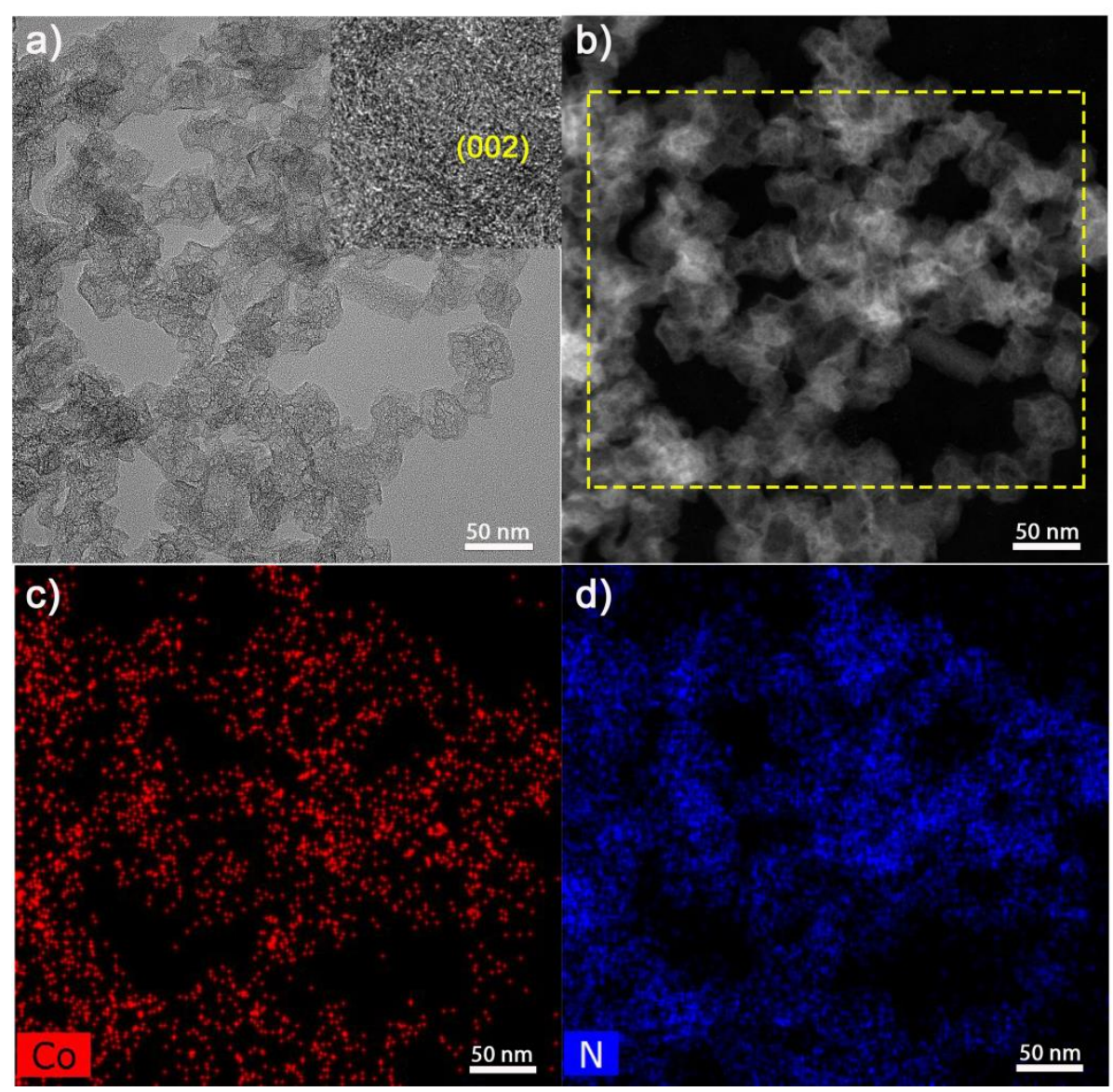

Figure 2: Bright field (a) and dark field (b) TEM image of Co@mesoNC catalyst; element mapping image of Co (c) and N (d) for Co@mesoNC catalyst.

uniformly dispersed Co and N EDX-signals can be clearly detected throughout the catalyst (Figure 2c,d). The atomic adsorption spectroscopy (AAS) and elemental analysis indicate that the Co@mesoNC catalyst has 3.5 wt.\% Co and 15.0 wt.\% N. At the same time, 2.8 wt.\% Zn and 1.6 wt.\% Si are also detected in Co@mesoNC, indicative of some residual Zn and Si in the sample even after high-temperature pyrolysis and $\mathrm{NaOH}$ washing. The high-resolution TEM (HR-TEM) image of Co@mesoNC in the insert of Figure 2a clearly demonstrates the presence of a layered graphitic carbon structure with a typical interplanar distance of $0.34 \mathrm{~nm}$. The absence of cobalt nanoparticles is further supported by powder XRD analysis (Figure 3a). No cobalt (oxide) reflections can be identified, and only two diffractions at $2 \theta=30^{\circ}$ and $50.5^{\circ}$ are observed, attributed to the (002) and (100) planes of the graphitic carbon, respectively [61]. The graphitization degree of the carbon matrix in the Co@mesoNC sample was further analyzed by Raman spectroscopy (Fig. S5). The characteristic D and G bands of 
carbon at $1350 \mathrm{~cm}^{-1}$ and $1580 \mathrm{~cm}^{-1}$ are clearly observed, correlated to graphitic disordered or defective carbon and $\mathrm{sp}^{2}$-bonded graphitic carbon sheets, respectively $[62,63]$. The BET area $\left(S_{B E T}\right)$ and pore volume $\left(V_{\text {pore }}\right)$ of the BIMZIF and Co@mesoNC were determined by $\mathrm{N}_{2}$ adsorption-desorption isotherms, as shown in Figure $3 \mathrm{~b}$ and Table S1. BIMZIF, with a high $S_{B E T}$ of $1780 \mathrm{~m}^{2} \mathrm{~g}^{-1}$ and $V_{\text {pore }}$ of $1.44 \mathrm{~cm}^{3} \mathrm{~g}^{-1}$, displays a steep $\mathrm{N}_{2}$ uptake at low relative pressures, typically associated with microporosity [50]. A hysteresis loop above $P / P_{0} \approx 0.8$ is also observed for BIMZIF, suggesting the presence of intergranular mesoporosity between the nanocrystals. After high-temperature pyrolysis and $\mathrm{SiO}_{2}$ leaching, $\mathrm{Co} @$ mesoNC exhibits a $S_{B E T}$ of $1230 \mathrm{~m}^{2} \mathrm{~g}^{-1}$ and $V_{\text {pore }}$ of $1.54 \mathrm{~cm}^{3} \mathrm{~g}^{-1}$. At the same time, the $\mathrm{N}_{2}$ adsorption-desorption isotherms of Co@mesoNC change to type IV with type $\mathrm{H}_{3}$ hysteresis that closes at $P / P_{0} \approx 0.4$, suggesting the presence of mesoporous structure inside the nanoparticles [64, 65].
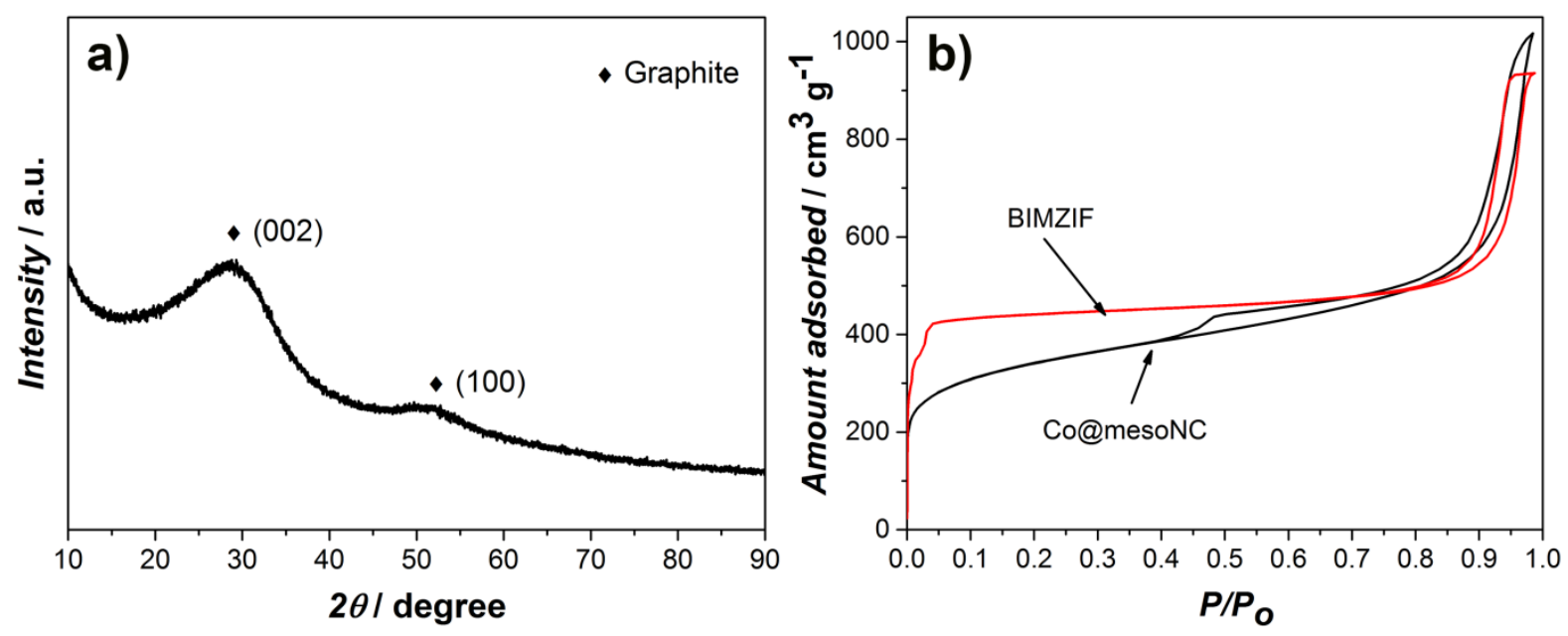

Figure 3: (a) XRD pattern of $\mathrm{Co@mesoNC}$ catalyst; (b) $\mathrm{N}_{2}$-adsorption-desorption isotherms of BIMZIF and Co@mesoNC materials.

The Co@mesoNC catalyst was further characterized by means of X-ray photoelectron spectroscopy (XPS). As shown in Figure 4a, N1s signals of Co@mesoNC can be described by the combination of four types of nitrogen species with binding energy around $398.5 \mathrm{eV}, 399.8$ $\mathrm{eV}, 400.8 \mathrm{eV}$, and $402.3 \mathrm{eV}$, which can be attributed to pyridinic-N (53.4 at.\%), pyrrolic-N (25.5 at.\%), quaternary-N (17.0 at.\%) and oxidized-N (4.1 at.\%), respectively [66, 67]. We speculate that, upon pyrolysis, $\mathrm{N}$ atoms in the pentagonal ring of the original imidazole units are mostly converted into pyridinic-, pyrrolic-, and quaternary-N species. The Co2p XPS spectrum of Co@mesoNC in Figure 4b highlights the absence of metallic Co (usually at 778.5 $\mathrm{eV})$, while the peak at $780.5 \mathrm{eV}$ can be ascribed to $\mathrm{Co}-\mathrm{N}_{\mathrm{x}}$ species $[53,61,68]$. 
Co K-edge X-ray absorption spectroscopy (XAS) measurements were carried out to further explore the local environment of cobalt in the Co@mesoNC sample. As shown in the X-ray absorption near-edge structure (XANES) spectrum (Figure 4c), a well-developed peak at $7709 \mathrm{eV}$ in the pre-edge region can be observed in the BIMZIF sample, arising from the electronic transition from the core $1 s$ to the unoccupied $3 d$ orbitals. This intense pre-edge peak clearly reveals that the local symmetry around cobalt ions is not centrosymmetric, confirming the tetrahedral symmetry around central cobalt atoms [69]. The edge energy $\left(E_{0}\right)$, located at $7717 \mathrm{eV}$, suggest the presence of divalent $\mathrm{Co}$ in the original framework. After pyrolysis and $\mathrm{SiO}_{2}$ removal, the edge for $\mathrm{Co} @ m e s o \mathrm{NC}$ is situated at $7720 \mathrm{eV}$ and is higher in energy to that of metallic Co in the pyrolyzed ZIF-67 at $7709 \mathrm{eV}$, indicating that no metallic cobalt phase is present in Co@mesoNC. The lower intensity of the pre-edge feature in Co@mesoNC compared to that of the original BIMZIF suggests that the tetrahedral coordination environment of cobalt in the framework changed during the high-temperature pyrolysis process. Similar shape, intensity and location of the pre-edge feature has been observed in case of quadrupole $1 s \rightarrow 3 d$ transition in distorted octahedral coordination environment [70-72]. The similar location of the white line (with a threshold energy of 7731 $\mathrm{eV}$ ) and the marked differences of the continuous resonance shape between both samples (BIMZIF and Co@mesoNC) also confirms a drastic change in the local geometry around cobalt [69].

Further local structural information can be obtained from extended X-ray absorption fine structure (EXAFS, Figure 4d). The fittings of the R-space curve for BIMZIF yields a first Co$\mathrm{N}$ coordination sphere at $1.99 \AA$ bond distance (inset of Figure 4d and Fig. S7c,d) [53, 69]. After pyrolysis, the first coordination sphere around Co shifts to a lower bond distance (1.89 $\AA$ ), demonstrating that the coordination of Co varies slightly. Importantly, in the fittings of the EXAFS signal for Co@mesoNC it was not possible to include any Co-Co scattering paths with reasonable parameters (Fig. S6 and Table S2). In contrast, the metallic Co nanoparticles in the pyrolyzed ZIF-67 (Fig. S7e,f and Table S2) clearly show exclusively the Co-Co path that can be fitted with Co-Co bond distances of $2.49 \AA$. These marked differences further confirm that Co is atomically dispersed in the Co@mesoNC sample.

To clarify the role of $\mathrm{SiO}_{2}$ during the synthesis, we prepared a sample, denoted as $\mathrm{Co@NC}$, by direct pyrolysis of BIMZIF. In the absence of $\mathrm{SiO}_{2}$ during pyrolysis, both cobalt nanoparticles and highly dispersed Co- $\mathrm{N}_{x}$ species can be observed in the TEM image of this sample (Fig. S8a-d), although it exhibits a comparable graphitization degree as the Co@mesoNC sample, as concluded from a similar $I_{D} / I_{G}$ ratio in the Raman spectra (Fig. S5). 
In addition, $\mathrm{N}_{2}$ adsorption-desorption isotherms indicate that $\mathrm{Co} @ \mathrm{NC}$ exhibits a typical microporous structure with some intergranular mesoporosity, as concluded from the presence of hysteresis loop above $P / P_{0} \approx 0.8$ (Fig. S9). The Brunauer-Emmett-Teller area $\left(S_{B E T}\right)$ and pore volume $\left(V_{\text {pore }}\right)$ of Co@NC are $680 \mathrm{~m}^{2} \mathrm{~g}^{-1}$ and $0.98 \mathrm{~m}^{3} \mathrm{~g}^{-1}$, much lower than that of Co@ mesoNC $\left(1230 \mathrm{~m}^{2} \mathrm{~g}^{-1}\right.$ and $\left.1.54 \mathrm{~m}^{3} \mathrm{~g}^{-1}\right)$ (Table $\left.\mathrm{S} 1\right)$, attributed to the collapse of the welldefined microporous structure of BIMZIF during the pyrolysis process [36, 73].
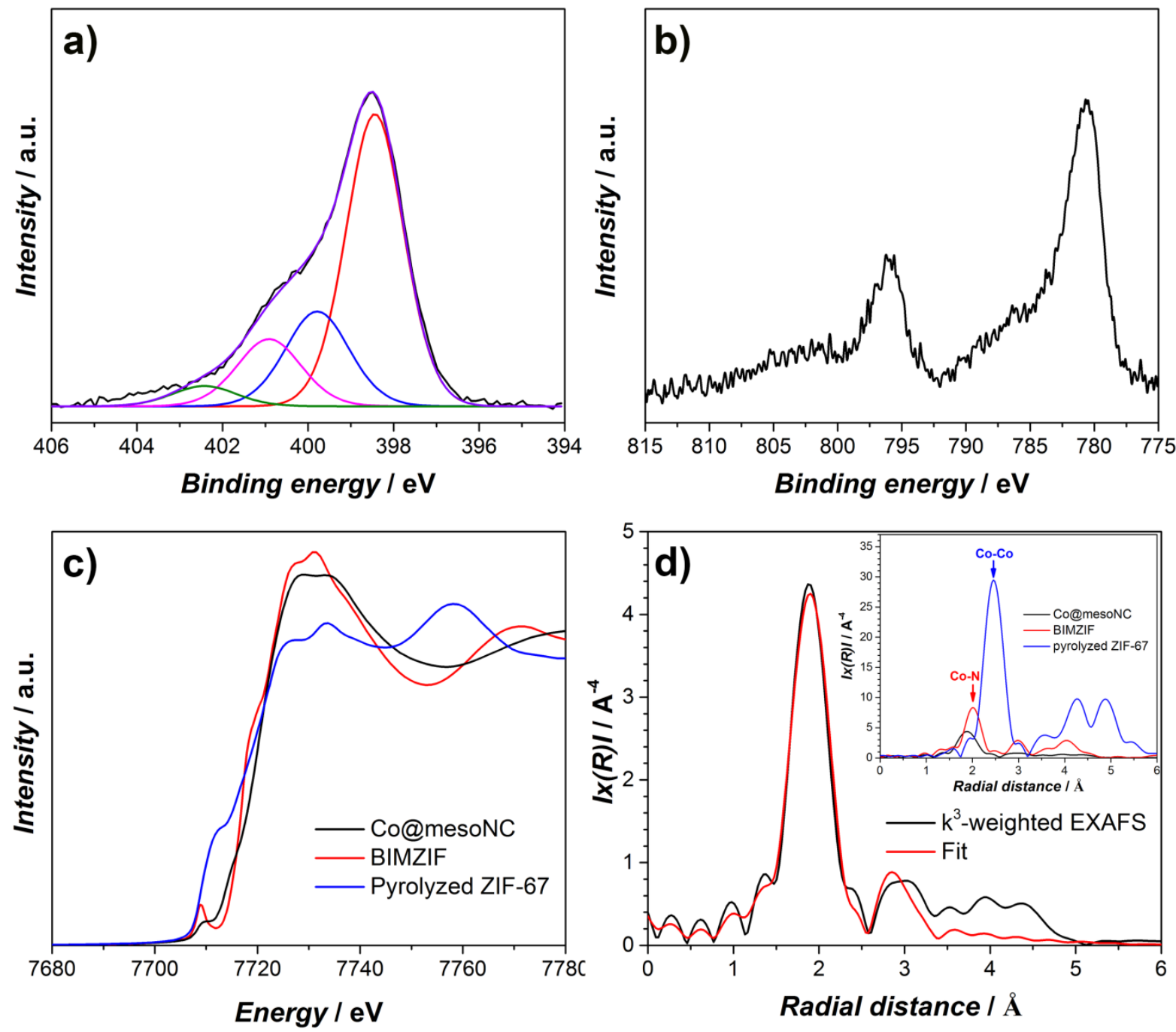

Figure 4: N1s (a) and Co2p (b) XPS regions of Co@mesoNC catalyst; (c) Co $K$-edge XANES spectra; and (d) Fitting of phase corrected $k^{3}$-weighted $\chi(k)$-function EXAFS spectra of Co@mesoNC catalyst, together with the phase corrected Co@mesoNC, BIMZIF and pyrolyzed ZIF-67 in the inset.

The preferred formation of Co- $\mathrm{N}_{\mathrm{x}}$ sites and the absence of cobalt nanoparticles in the Co@mesoNC catalyst can be attributed to the following: (i) When a large amount of $\mathrm{Zn}$ is present in the original BIMZIF material, the spatial distance between two Co atoms can be finely controlled by $\mathrm{Zn}$ atoms acting as a spacer, thereby precluding the agglomeration of 
cobalt atoms to nanoparticles during the high-temperature pyrolysis; (ii) During the pyrolysis process, $\mathrm{Zn}$ evaporate [74], and free $\mathrm{N}$-coordination sites can be generated in the carbon matrix, which help stabilize the Co atoms $[52,53]$ and (iii) The presence of $\mathrm{SiO}_{2}$ nanoparticles in the well-developed porosity of BIMZIF further mitigates the sintering of Co atoms under high-temperature pyrolysis condition [75]. Thus, the $\mathrm{SiO}_{2}$-templated strategy in this work impedes the formation of cobalt nanoparticles, creates mesoporosity and maintains a high specific surface area in the N-doped carbon matrix.

Table 1: Results of the catalytic hydrogenation of nitrobenzene over the prepared catalysts a.

\begin{tabular}{|c|c|c|c|c|c|c|c|}
\hline Entry & Sample & $\begin{array}{c}T \\
(\mathrm{~K})\end{array}$ & $\begin{array}{c}P \\
(\mathrm{MPa})\end{array}$ & $\begin{array}{c}X_{\text {Nitro }} \\
(\%)\end{array}$ & $\begin{array}{l}S_{\text {Aniline }} \\
(\%)\end{array}$ & $T O F^{\mathrm{b}}$ & $\begin{array}{l}\text { Catalyst productivity } \\
\left(\mathrm{mmol}_{\mathrm{NB}} \mathrm{h}^{-1} \mathrm{mg}_{\mathrm{Co}}{ }^{-1}\right)^{\mathrm{c}}\end{array}$ \\
\hline 1 & No catalyst & 383 & 3 & - & - & - & - \\
\hline $2^{c}$ & Co@mesoNC & 383 & 3 & - & - & - & - \\
\hline 3 & BIMZIF & 383 & 3 & - & - & - & - \\
\hline $4^{\mathrm{d}}$ & $\mathrm{NC}$ & 383 & 3 & - & - & - & - \\
\hline 5 & Co@mesoNC & 383 & 3 & 55 & $>99$ & 42 & 0.72 \\
\hline 6 & Co@mesoNC & 383 & 0.5 & 14 & $>99$ & 11 & 0.18 \\
\hline 7 & Co@mesoNC & 383 & 1 & 25 & $>99$ & 19 & 0.32 \\
\hline 8 & Co@mesoNC & 383 & 2 & 43 & $>99$ & 33 & 0.55 \\
\hline 9 & Co@mesoNC & 343 & 3 & 12 & $>99$ & 9 & 0.15 \\
\hline 10 & Co@mesoNC & 363 & 3 & 27 & $>99$ & 21 & 0.34 \\
\hline
\end{tabular}

a: Reaction conditions: $1 \mathrm{mmol}$ nitrobenzene, $11 \mathrm{mg}$ catalyst, substrate to cobalt molar ratio of $150,0.65 \mathrm{mmol}$ dodecane as internal standard, $5 \mathrm{~mL}$ ethanol, $383 \mathrm{~K}, 3 \mathrm{MPa} \mathrm{H}_{2}, 2 \mathrm{~h} .{ }^{\text {b: }}: T O F=$ moles of converted substrate per

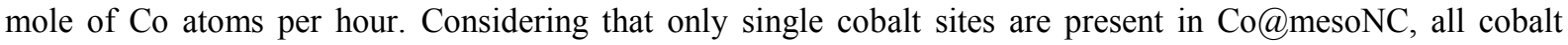
atoms participate in the reaction. ${ }^{\mathrm{c}}$ : calculated by moles of nitrobenzene consumed divided by total weight of cobalt per hour. ${ }^{\mathrm{c}}$ : reaction was performed in He atmosphere. d: prepared by pyrolysis of ZIF-8 crystals under $\mathrm{N}_{2}$ flow.

\subsection{Catalytic Hydrogenation of Nitrobenzene to Aniline over the Co@mesoNC catalyst}

Direct hydrogenation of nitrobenzene with $\mathrm{H}_{2}$ requires an efficient catalyst to simultaneously promote the activation of hydrogen and nitro groups in the substrates. In order to clearly understand the catalytic activity of the $\mathrm{Co} @$ mesoNC catalyst in this process, a series of control experiments were firstly performed. As shown in Table 1, hydrogenation of nitrobenzene to aniline did not proceed in the absence of catalyst (Entry 1, Table 1) or $\mathrm{H}_{2}$ (Entry 2, Table 1). Similarly, no aniline was detected over the original BIMZIF (Entry 3, Table 1) and the 'NC' sample prepared by pyrolysis of ZIF-8 (Entry 4, Table 1) under the same conditions. However, the Co@mesoNC sample efficiently catalyzed the hydrogenation of nitrobenzene to aniline without any other detectable products (Entry 5, Table 1). These results reveal that the highly dispersed Co- $\mathrm{N}_{\mathrm{x}}$ species generated during high temperature pyrolysis are the active sites for the chemoselective hydrogenation of nitro groups. In addition, 
the hydrogenation activity of the Co@mesoNC catalyst is dependent on $\mathrm{H}_{2}$ pressure (Entries 5-8, Table 1) and reaction temperature (Entry 5, Entries 9-10, Table 1). Obviously, a higher $\mathrm{H}_{2}$ pressure and a high reaction temperature promote the hydrogenation; hence in all subsequent experiments the reaction conditions were fixed at $3 \mathrm{MPa} \mathrm{H}_{2}$ and $383 \mathrm{~K}$. Importantly, the kinetic profile of the reaction in Figure 5a indicates that nitrobenzene could be converted smoothly into aniline with initially a nearly zero order in concentration, and no intermediates could be detected by gas chromatography during the entire reaction process. Interestingly, the catalytic activity of $\mathrm{Co@mesoNC} \mathrm{is} \mathrm{superior} \mathrm{to} \mathrm{the} \mathrm{behavior} \mathrm{of} \mathrm{other}$ recently reported supported cobalt catalysts (Table S3), such as Co@N-doped carbon [29], $\mathrm{CoO}_{\mathrm{x}} @ \mathrm{NCNTs}$ [30], Co-SiCN [31], and Co-Co $\mathrm{O}_{4} / \mathrm{NGr} @ \mathrm{C}$ [42].
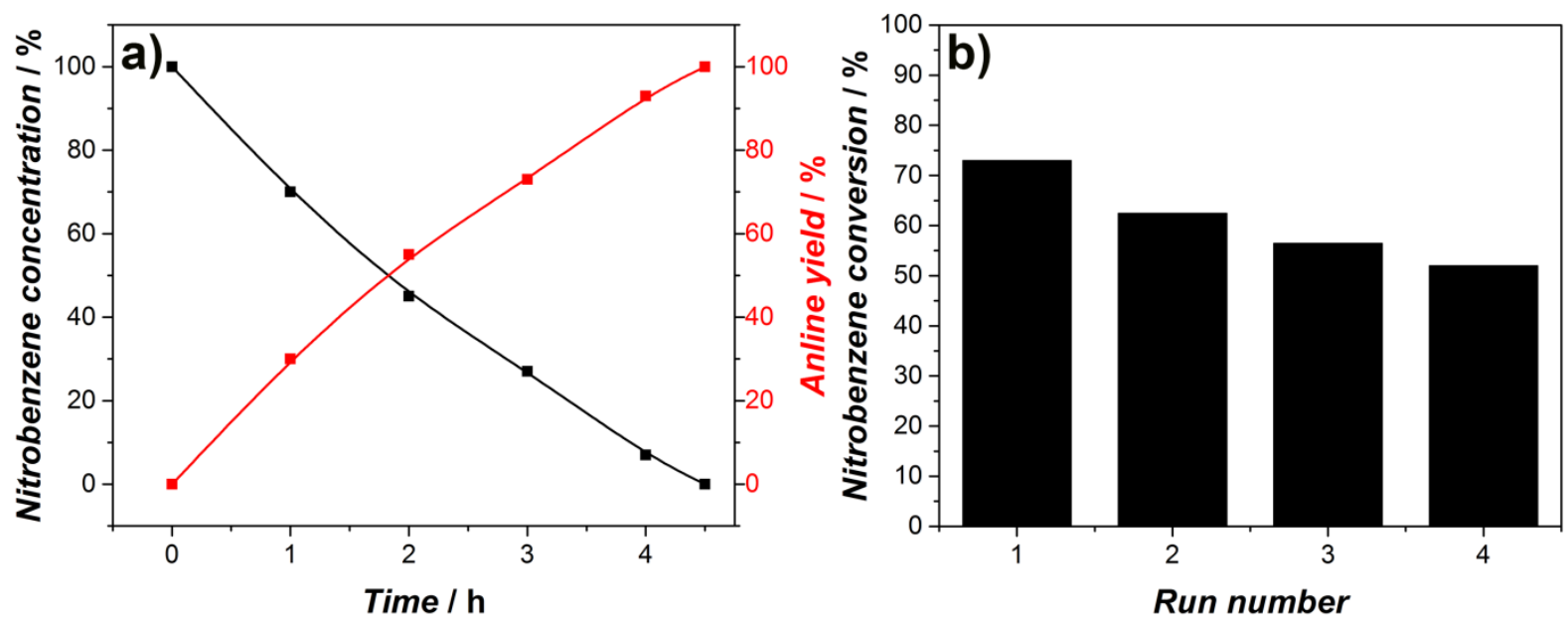

Figure 5: (a) Kinetic data of hydrogenation of nitrobenzene with Co@mesoNC catalyst. The plot is based on the results of different reactions stopped at different times. (b) Recycling experiments of hydrogenation of nitrobenzene with Co@mesoNC catalyst. Reaction conditions: 1 mmol nitrobenzene, $11 \mathrm{mg}$ catalyst, substrate to cobalt molar ratio of $150,0.65 \mathrm{mmol}$ dodecane as internal standard, $5 \mathrm{~mL}$ ethanol, $383 \mathrm{~K}, 3 \mathrm{MPa} \mathrm{H}_{2}, 3 \mathrm{~h}$ for recycling experiments. Aniline selectivity $>99 \%$.

The stability and reusability of the Co@mesoNC catalyst was also investigated. As presented in Figure 5b, under the studied conditions, the Co@mesoNC catalyst exhibits a nitrobenzene conversion of $73 \%$ after $3 \mathrm{~h}$, and can be reused at least four times with a nitrobenzene conversion still above 51\% after the fourth run. The TEM image of the spent Co@mesoNC after the fourth run shows that the Co atoms in the N-doped carbon matrix are still well dispersed and no cobalt nanoparticles can be detected (Fig. S10), excluding the agglomeration of cobalt as a major cause of deactivation during hydrogenation. The structure of the spent Co@mesoNC catalyst is still well preserved as revealed by the Raman spectrum in Fig. S11 and XPS analysis in Fig. S12, suggesting that the deactivation cannot be attributed 
to any modification in the carbon structure during the hydrogenation process. ICP analysis of the reaction mixture indicates a small amount of cobalt ( $3 \mathrm{wt} . \%$ of the total cobalt content) in solution after each run (Table S4). At the same time, after each run, a slight increase of catalyst mass $(\sim 2 \%)$ was observed even after a thorough washing with ethanol and vacuum drying, indicative of some residual deposit in the pores of the catalysts, which cannot be easily removed. Hence, the deactivation of Co@mesoNC catalyst is ascribed mainly to fouling by unidentified deposits during reaction, although some minute loss of cobalt cannot be excluded. This fouling is stronger for the microporous Co@NC sample prepared by direct pyrolysis of BIMZIF(Co,Zn). After an initial nitrobenzene conversion of only $34 \%$, this decreases sharply to $6 \%$ after 4 consecutive runs (Fig. S13) under similar reaction conditions. The inferior hydrogenation performance of $\mathrm{Co@NC} \mathrm{further} \mathrm{demonstrates} \mathrm{the} \mathrm{high} \mathrm{activity} \mathrm{of}$ these highly dispersed $\mathrm{Co}-\mathrm{N}_{\mathrm{x}}$ sites in the mesoporous carbon matrix, which contributes to the accessibility of the active Co- $\mathrm{N}_{\mathrm{x}}$ sites and improved mass transport of substrate molecules during the hydrogenation process.

\subsection{Co@mesoNC Catalyzed Hydrogenation of Nitroarenes to Substituted Anilines}

To explore the general applicability of Co@mesoNC, a variety of substituted nitroarenes containing electron-donating and electron-withdrawing groups at different positions were investigated under the standard test conditions. As shown in Table 2, the Co@mesoNC catalyst exhibits a high activity for the hydrogenation of industrially relevant nitroarenes such as fluoro- and chloro-nitrobenzenes toward their corresponding anilines in high selectivities (Table 2, entries 1-4). Even a sterically hindered nitroarene was hydrogenated to the substituted aniline with outstanding activity and chemoselectivity (Table 2, entry 5). As it is well known, the most-challenging substrates are those that bear other easily reducible groups (Table 2, entries 6-10). Like other N-doped carbon supported metal catalysts prepared using high-temperature pyrolysis strategy [29-32, 36-39, 42], the Co@mesoNC catalyst is able to chemoselectively reduce nitro groups in the presence of $\mathrm{C}=\mathrm{C}$ bonds with high activity (Table 2, entry 6 and Table S5), which is hardly achieved by Pt-group and Ni catalysts [39, 42]. Furthermore, substrates containing sensitive groups, such as $-\mathrm{I},-\mathrm{OCH}_{3}$, and $-\mathrm{COCH}_{3}$, can be as well smoothly and chemoselectively hydrogenated into the corresponding anilines without dehalogenation and/or competitive hydrogenation on these substituted moieties (Table 2, entries 7-10). All these results demonstrate again that the atomically dispersed $\mathrm{Co}-\mathrm{N}_{\mathrm{x}}$ sites in the mesoporous N-doped carbon matrix displays excellent catalytic activity and chemoselectivity for hydrogenation of nitroarenes using molecular hydrogen. 
Table 2: Results of the chemoselective hydrogenation of substituted nitroarenes over Co@mesoNC a .

\begin{tabular}{|c|c|c|c|c|}
\hline Entry & Substrate & Product & $S(\%)^{\mathrm{b}}$ & $\begin{array}{l}\text { Catalyst productivity } \\
\left(\mathrm{mmol}_{\text {Nitro }} \mathrm{h}^{-1} \mathrm{mg}_{\mathrm{Co}^{-1}}\right)^{\mathrm{c}}\end{array}$ \\
\hline 1 & & & $>98$ & 0.70 \\
\hline 2 & & & $>99$ & 0.79 \\
\hline 3 & & & $>99$ & 0.79 \\
\hline 4 & & & $>98$ & 0.75 \\
\hline 5 & & & $>99$ & 0.40 \\
\hline 6 & & & $>97$ & 0.46 \\
\hline 7 & & & $>97$ & 0.22 \\
\hline 8 & & & $>98$ & 0.53 \\
\hline $9^{d}$ & & & $>93$ & 0.51 \\
\hline $10^{\mathrm{d}}$ & & & $>96$ & 0.48 \\
\hline
\end{tabular}

a: Reaction conditions: $1 \mathrm{mmol}$ nitrobenzene, $11 \mathrm{mg}$ catalyst, substrate to cobalt molar ratio of 150, $0.65 \mathrm{mmol}$ dodecane as internal standard, $5 \mathrm{~mL}$ ethanol, $383 \mathrm{~K}, 3 \mathrm{MPa} \mathrm{H}_{2}, 2 \mathrm{~h}$; b: selectivity to the substituted anilines; c: calculated by moles of nitroarenes consumed divided by total mass of cobalt per hour; d: reaction time $3 \mathrm{~h}$.

\section{CONCLUSIONS}

In this work, a strategy is presented to synthesize a supported cobalt catalyst with atomically dispersed $\mathrm{Co}-\mathrm{N}_{\mathrm{x}}$ sites $(3.5 \mathrm{wt} . \% \mathrm{Co})$ in a mesoporous $\mathrm{N}$-doped carbon matrix. The synthesis process consists of hydrolysis of TMOS molecules in BIMZIF(Co,Zn), high- 
temperature pyrolysis and removal of the $\mathrm{SiO}_{2}$ template. The preferential formation and high stability of these Co- $\mathrm{N}_{\mathrm{x}}$ sites in the carbon support are attributed to the presence of a large amount of $\mathrm{Zn}$ and $\mathrm{N}$ in the structure of the BIMZIF precursor and the presence of $\mathrm{SiO}_{2}$ in the porosity of BIMZIF, which, to a large extent, effectively separates cobalt atoms, thereby impeding their agglomeration to nanoparticles. The $\mathrm{SiO}_{2}$-templated approach is proven essential to generate mesoporosity in the N-doped carbon support and to maintain a high specific surface area in the carbon support during high-temperature pyrolysis as well. These Co- $\mathrm{N}_{\mathrm{x}}$ sites in the mesoporous $\mathrm{N}$-doped carbon matrix exhibit an outstanding activity and selectivity for the chemoselective hydrogenation of nitroarenes for a wide range of substrates under mild reaction conditions. The mesoporosity of this catalyst probably guaranteed a good mass transport of reactants and/or products and the accessibility of the active Co- $\mathrm{N}_{\mathrm{x}}$ sites, thereby also reducing the deactivation of the catalyst by fouling.

\section{ACKNOWLEDGMENTS}

We thank for the support from the Dutch-Belgian program for access to the BM26A in ESRF, Grenoble. We thank A. Dikhtiarenko for help in the design of the graphical abstract. Xiaohui Sun acknowledges financial support from China Scholarship Council (CSC).

\section{REFERENCES}

[1] R.S. Downing, P.J. Kunkeler, H. van Bekkum, Catalytic Amination ReactionsCatalytic syntheses of aromatic amines, Catalysis Today, 37 (1997) 121-136.

[2] N. Ono, The Nitro Group in Organic Synthesis, Wiley-VCH, New York, 2001.

[3] H.-U. Blaser, H. Steiner, M. Studer, Selective Catalytic Hydrogenation of Functionalized Nitroarenes: An Update, ChemCatChem, 1 (2009) 210-221.

[4] H.-U. Blaser, C. Malan, B. Pugin, F. Spindler, H. Steiner, M. Studer, Selective Hydrogenation for Fine Chemicals: Recent Trends and New Developments, Advanced Synthesis \& Catalysis, 345 (2003) 103-151.

[5] P.N. Rylander, Catalytic Hydrogenation in Organic Syntheses: Paul Rylander, Academic Press Incorporated, 1979.

[6] J.G. de Vries, C.J. Elsevier, The handbook of homogeneous hydrogenation, Wiley-VCH, 2007.

[7] P.G. Andersson, I.J. Munslow, Modern Reduction Methods, Wiley, 2008.

[8] F. L'Eplattenier, P. Matthys, F. Calderazzo, Homogeneous ruthenium-catalyzed reduction of nitrobenzene, Inorganic Chemistry, 9 (1970) 342-345.

[9] A. Corma, C. González-Arellano, M. Iglesias, F. Sánchez, Gold complexes as catalysts: Chemoselective hydrogenation of nitroarenes, Applied Catalysis A: General, 356 (2009) 99102.

[10] K. Junge, K. Schroder, M. Beller, Homogeneous catalysis using iron complexes: recent developments in selective reductions, Chemical Communications, 47 (2011) 4849-4859. 
[11] R.J. Rahaim, R.E. Maleczka, Pd-Catalyzed Silicon Hydride Reductions of Aromatic and Aliphatic Nitro Groups, Organic Letters, 7 (2005) 5087-5090.

[12] P.M. Reis, B. Royo, Chemoselective hydrogenation of nitroarenes and deoxygenation of pyridine N-oxides with $\mathrm{H} 2$ catalyzed by $\mathrm{MoO} 2 \mathrm{Cl} 2$, Tetrahedron Letters, 50 (2009) 949-952.

[13] S. Nishimura, Handbook of Heterogeneous Catalytic Hydrogenation for Organic Synthesis, Wiley, 2001.

[14] R.A. Sheldon, H. Bekkum, Fine Chemicals Through Heterogenous Catalysis, WileyVCH, 2001.

[15] R.V. Jagadeesh, A.-E. Surkus, H. Junge, M.-M. Pohl, J. Radnik, J. Rabeah, H. Huan, V. Schünemann, A. Brückner, M. Beller, Nanoscale Fe2O3-Based Catalysts for Selective Hydrogenation of Nitroarenes to Anilines, Science, 342 (2013) 1073-1076.

[16] A. Corma, P. Serna, Chemoselective Hydrogenation of Nitro Compounds with Supported Gold Catalysts, Science, 313 (2006) 332-334.

[17] Y. Chen, C. Wang, H. Liu, J. Qiu, X. Bao, Ag/SiO2: a novel catalyst with high activity and selectivity for hydrogenation of chloronitrobenzenes, Chemical Communications, (2005) 5298-5300.

[18] A. Onopchenko, E.T. Sabourin, C.M. Selwitz, Selective catalytic hydrogenation of aromatic nitro groups in the presence of acetylenes. Synthesis of (3-aminophenyl)acetylene via hydrogenation of dimethylcarbinol substituted (3-nitrophenyl)acetylene over heterogeneous metallic ruthenium catalyst, The Journal of Organic Chemistry, 44 (1979) 1233-1236.

[19] G. Vilé, N. Almora-Barrios, N. López, J. Pérez-Ramírez, Structure and Reactivity of Supported Hybrid Platinum Nanoparticles for the Flow Hydrogenation of Functionalized Nitroaromatics, ACS Catalysis, 5 (2015) 3767-3778.

[20] A. Yarulin, C. Berguerand, I. Yuranov, F. Cárdenas-Lizana, I. Prokopyeva, L. KiwiMinsker, $\mathrm{Pt}-\mathrm{Zn}$ nanoparticles supported on porous polymeric matrix for selective 3nitrostyrene hydrogenation, Journal of Catalysis, 321 (2015) 7-12.

[21] M. Carrus, M. Fantauzzi, F. Riboni, M. Makosch, A. Rossi, E. Selli, J.A. van Bokhoven, Increased conversion and selectivity of 4-nitrostyrene hydrogenation to 4-aminostyrene on $\mathrm{Pt}$ nanoparticles supported on titanium-tungsten mixed oxides, Applied Catalysis A: General, 519 (2016) 130-138.

[22] J. Gu, Z. Zhang, P. Hu, L. Ding, N. Xue, L. Peng, X. Guo, M. Lin, W. Ding, Platinum Nanoparticles Encapsulated in MFI Zeolite Crystals by a Two-Step Dry Gel Conversion Method as a Highly Selective Hydrogenation Catalyst, ACS Catalysis, 5 (2015) 6893-6901.

[23] S. Zhang, C.-R. Chang, Z.-Q. Huang, J. Li, Z. Wu, Y. Ma, Z. Zhang, Y. Wang, Y. Qu, High Catalytic Activity and Chemoselectivity of Sub-nanometric Pd Clusters on Porous Nanorods of $\mathrm{CeO} 2$ for Hydrogenation of Nitroarenes, Journal of the American Chemical Society, 138 (2016) 2629-2637.

[24] A. Corma, P. Serna, P. Concepción, J.J. Calvino, Transforming Nonselective into Chemoselective Metal Catalysts for the Hydrogenation of Substituted Nitroaromatics, Journal of the American Chemical Society, 130 (2008) 8748-8753.

[25] G.C. Bond, Supported metal catalysts: some unsolved problems, Chemical Society Reviews, 20 (1991) 441-475.

[26] B. Coq, A. Tijani, R. Dutartre, F. Figuéras, Influence of support and metallic precursor on the hydrogenation of p-chloronitrobenzene over supported platinum catalysts, Journal of Molecular Catalysis, 79 (1993) 253-264.

[27] X. Han, R. Zhou, G. Lai, X. Zheng, Influence of support and transition metal (Cr, Mn, $\mathrm{Fe}, \mathrm{Co}, \mathrm{Ni}$ and $\mathrm{Cu}$ ) on the hydrogenation of $\mathrm{p}$-chloronitrobenzene over supported platinum catalysts, Catalysis Today, 93-95 (2004) 433-437. 
[28] S. Kataoka, Y. Takeuchi, A. Harada, T. Takagi, Y. Takenaka, N. Fukaya, H. Yasuda, T. Ohmori, A. Endo, Microreactor containing platinum nanoparticles for nitrobenzene hydrogenation, Applied Catalysis A: General, 427-428 (2012) 119-124.

[29] D. Formenti, F. Ferretti, C. Topf, A.-E. Surkus, M.-M. Pohl, J. Radnik, M. Schneider, K. Junge, M. Beller, F. Ragaini, Co-based heterogeneous catalysts from well-defined $\alpha$-diimine complexes: Discussing the role of nitrogen, Journal of Catalysis, 351 (2017) 79-89.

[30] Z. Wei, J. Wang, S. Mao, D. Su, H. Jin, Y. Wang, F. Xu, H. Li, Y. Wang, In SituGenerated Co0-Co3O4/N-Doped Carbon Nanotubes Hybrids as Efficient and Chemoselective Catalysts for Hydrogenation of Nitroarenes, ACS Catalysis, 5 (2015) 4783-4789.

[31] T. Schwob, R. Kempe, A Reusable Co Catalyst for the Selective Hydrogenation of Functionalized Nitroarenes and the Direct Synthesis of Imines and Benzimidazoles from Nitroarenes and Aldehydes, Angewandte Chemie International Edition, 55 (2016) 1517515179.

[32] T. Cheng, H. Yu, F. Peng, H. Wang, B. Zhang, D. Su, Identifying active sites of CoNC/CNT from pyrolysis of molecularly defined complexes for oxidative esterification and hydrogenation reactions, Catalysis Science \& Technology, 6 (2016) 1007-1015.

[33] B. Chen, F. Li, Z. Huang, G. Yuan, Recyclable and Selective Nitroarene Hydrogenation Catalysts Based on Carbon-Coated Cobalt Oxide Nanoparticles, ChemCatChem, 8 (2016) 1132-1138.

[34] G. Hahn, J.-K. Ewert, C. Denner, D. Tilgner, R. Kempe, A Reusable Mesoporous Nickel Nanocomposite Catalyst for the Selective Hydrogenation of Nitroarenes in the Presence of Sensitive Functional Groups, ChemCatChem, 8 (2016) 2461-2465.

[35] S. Pisiewicz, D. Formenti, A.-E. Surkus, M.-M. Pohl, J. Radnik, K. Junge, C. Topf, S. Bachmann, M. Scalone, M. Beller, Synthesis of Nickel Nanoparticles with N-Doped Graphene Shells for Catalytic Reduction Reactions, ChemCatChem, 8 (2016) 129-134.

[36] X. Sun, A.I. Olivos-Suarez, L. Oar-Arteta, E. Rozhko, D. Osadchii, A. Bavykina, F. Kapteijn, J. Gascon, Metal-Organic Framework Mediated Cobalt/Nitrogen-Doped Carbon Hybrids as Efficient and Chemoselective Catalysts for the Hydrogenation of Nitroarenes, ChemCatChem, 9 (2017) 1854-1862.

[37] W.-J. Liu, K. Tian, H. Jiang, One-pot synthesis of Ni-NiFe2O4/carbon nanofiber composites from biomass for selective hydrogenation of aromatic nitro compounds, Green Chemistry, 17 (2015) 821-826.

[38] L. Liu, P. Concepción, A. Corma, Non-noble metal catalysts for hydrogenation: A facile method for preparing Co nanoparticles covered with thin layered carbon, Journal of Catalysis, 340 (2016) $1-9$.

[39] L. Liu, F. Gao, P. Concepción, A. Corma, A new strategy to transform mono and bimetallic non-noble metal nanoparticles into highly active and chemoselective hydrogenation catalysts, Journal of Catalysis, 350 (2017) 218-225.

[40] F. Zhang, C. Zhao, S. Chen, H. Li, H. Yang, X.-M. Zhang, In situ mosaic strategy generated Co-based $\mathrm{N}$-doped mesoporous carbon for highly selective hydrogenation of nitroaromatics, Journal of Catalysis, 348 (2017) 212-222.

[41] X. Wang, Y. Li, Chemoselective hydrogenation of functionalized nitroarenes using MOF-derived co-based catalysts, Journal of Molecular Catalysis A: Chemical, 420 (2016) 5665.

[42] F.A. Westerhaus, R.V. Jagadeesh, G. Wienhöfer, M.-M. Pohl, J. Radnik, A.-E. Surkus, J. Rabeah, K. Junge, H. Junge, M. Nielsen, A. Brückner, M. Beller, Heterogenized cobalt oxide catalysts for nitroarene reduction by pyrolysis of molecularly defined complexes, Nat Chem, 5 (2013) 537-543. 
[43] R.V. Jagadeesh, D. Banerjee, P.B. Arockiam, H. Junge, K. Junge, M.-M. Pohl, J. Radnik, A. Bruckner, M. Beller, Highly selective transfer hydrogenation of functionalised nitroarenes using cobalt-based nanocatalysts, Green Chemistry, 17 (2015) 898-902.

[44] D. Formenti, C. Topf, K. Junge, F. Ragaini, M. Beller, Fe2O3/NGr@C- and CoCo3O4/NGr@C-catalysed hydrogenation of nitroarenes under mild conditions, Catalysis Science \& Technology, 6 (2016) 4473-4477.

[45] K. Shen, L. Chen, J. Long, W. Zhong, Y. Li, MOFs-Templated Co@Pd Core-Shell NPs Embedded in N-Doped Carbon Matrix with Superior Hydrogenation Activities, ACS Catalysis, 5 (2015) 5264-5271.

[46] P. Zhou, L. Jiang, F. Wang, K. Deng, K. Lv, Z. Zhang, High performance of a cobaltnitrogen complex for the reduction and reductive coupling of nitro compounds into amines and their derivatives, Science Advances, 3 (2017).

[47] X.-C. Huang, Y.-Y. Lin, J.-P. Zhang, X.-M. Chen, Ligand-Directed Strategy for ZeoliteType Metal-Organic Frameworks: Zinc(II) Imidazolates with Unusual Zeolitic Topologies, Angewandte Chemie International Edition, 45 (2006) 1557-1559.

[48] K.S. Park, Z. Ni, A.P. Côté, J.Y. Choi, R. Huang, F.J. Uribe-Romo, H.K. Chae, M. O'Keeffe, O.M. Yaghi, Exceptional chemical and thermal stability of zeolitic imidazolate frameworks, Proceedings of the National Academy of Sciences, 103 (2006) 10186-10191.

[49] R. Banerjee, A. Phan, B. Wang, C. Knobler, H. Furukawa, M. O'Keeffe, O.M. Yaghi, High-Throughput Synthesis of Zeolitic Imidazolate Frameworks and Application to $\mathrm{CO} 2$ Capture, Science, 319 (2008) 939-943.

[50] J. Tang, R.R. Salunkhe, J. Liu, N.L. Torad, M. Imura, S. Furukawa, Y. Yamauchi, Thermal Conversion of Core-Shell Metal-Organic Frameworks: A New Method for Selectively Functionalized Nanoporous Hybrid Carbon, Journal of the American Chemical Society, 137 (2015) 1572-1580.

[51] J. Yang, F. Zhang, H. Lu, X. Hong, H. Jiang, Y. Wu, Y. Li, Hollow Zn/Co ZIF Particles Derived from Core-Shell ZIF-67@ZIF-8 as Selective Catalyst for the Semi-Hydrogenation of Acetylene, Angewandte Chemie International Edition, 54 (2015) 10889-10893.

[52] Y.-Z. Chen, C. Wang, Z.-Y. Wu, Y. Xiong, Q. Xu, S.-H. Yu, H.-L. Jiang, From Bimetallic Metal-Organic Framework to Porous Carbon: High Surface Area and Multicomponent Active Dopants for Excellent Electrocatalysis, Advanced Materials, 27 (2015) 5010-5016.

[53] P. Yin, T. Yao, Y. Wu, L. Zheng, Y. Lin, W. Liu, H. Ju, J. Zhu, X. Hong, Z. Deng, G. Zhou, S. Wei, Y. Li, Single Cobalt Atoms with Precise N-Coordination as Superior Oxygen Reduction Reaction Catalysts, Angewandte Chemie International Edition, 55 (2016) 1080010805.

[54] J. Tang, R.R. Salunkhe, H. Zhang, V. Malgras, T. Ahamad, S.M. Alshehri, N. Kobayashi, S. Tominaka, Y. Ide, J.H. Kim, Y. Yamauchi, Bimetallic Metal-Organic Frameworks for Controlled Catalytic Graphitization of Nanoporous Carbons, Scientific Reports, 6 (2016) 30295.

[55] X. Kang, H. Liu, M. Hou, X. Sun, H. Han, T. Jiang, Z. Zhang, B. Han, Synthesis of Supported Ultrafine Non-noble Subnanometer-Scale Metal Particles Derived from MetalOrganic Frameworks as Highly Efficient Heterogeneous Catalysts, Angewandte Chemie, 128 (2016) 1092-1096.

[56] H. Li, H. Yang, H. Li, Highly active mesoporous Co-B amorphous alloy catalyst for cinnamaldehyde hydrogenation to cinnamyl alcohol, Journal of Catalysis, 251 (2007) 233238.

[57] M.F. De Lange, T.J.H. Vlugt, J. Gascon, F. Kapteijn, Adsorptive characterization of porous solids: Error analysis guides the way, Microporous and Mesoporous Materials, 200 (2014) 199-215. 
[58] L.A. Grunes, Study of the K edges of $3 d$ transition metals in pure and oxide form by $x-$ ray-absorption spectroscopy, Physical Review B, 27 (1983) 2111-2131.

[59] B. Ravel, M. Newville, ATHENA, ARTEMIS, HEPHAESTUS: data analysis for X-ray absorption spectroscopy using IFEFFIT, Journal of Synchrotron Radiation, 12 (2005) 537541.

[60] B. Ravel, M. Newville, ATHENA,ARTEMIS,HEPHAESTUS: data analysis for X-ray absorption spectroscopy usingIFEFFIT, J. Synchrotron Radiat., 12 (2005) 537-541.

[61] H. Fei, J. Dong, M.J. Arellano-Jiménez, G. Ye, N. Dong Kim, E.L.G. Samuel, Z. Peng, Z. Zhu, F. Qin, J. Bao, M.J. Yacaman, P.M. Ajayan, D. Chen, J.M. Tour, Atomic cobalt on nitrogen-doped graphene for hydrogen generation, Nature Communications, 6 (2015) 8668.

[62] B.Y. Xia, Y. Yan, N. Li, H.B. Wu, X.W. Lou, X. Wang, A metal-organic frameworkderived bifunctional oxygen electrocatalyst, Nature Energy, 1 (2016) 15006.

[63] P. Zhou, Z. Zhang, One-pot Reductive Amination of carbonyl Compounds with Nitro Compounds by Transfer Hydrogenation over Co-Nx as catalyst, ChemSusChem, 10 (2017) 1892-1897.

[64] Q. Wang, X. Qiu, W. Hu, Y. Huang, Facile synthesis of three-dimensional porous nitrogen doped carbon supported $\mathrm{Co} 3 \mathrm{O} 4$ for oxygen reduction reaction and oxygen evolution reaction, Materials Letters, 190 (2017) 169-172.

[65] P. Zhou, Z. Zhang, L. Jiang, C. Yu, K. Lv, J. Sun, S. Wang, A versatile cobalt catalyst for the reductive amination of carbonyl compounds with nitro compounds by transfer hydrogenation, Applied Catalysis B: Environmental, 210 (2017) 522-532.

[66] J.R. Pels, F. Kapteijn, J.A. Moulijn, Q. Zhu, K.M. Thomas, Evolution of nitrogen functionalities in carbonaceous materials during pyrolysis, Carbon, 33 (1995) 1641-1653.

[67] J. Casanovas, J.M. Ricart, J. Rubio, F. Illas, J.M. Jiménez-Mateos, Origin of the Large N 1s Binding Energy in X-ray Photoelectron Spectra of Calcined Carbonaceous Materials, Journal of the American Chemical Society, 118 (1996) 8071-8076.

[68] L. Shang, H. Yu, X. Huang, T. Bian, R. Shi, Y. Zhao, G.I.N. Waterhouse, L.-Z. Wu, C.H. Tung, T. Zhang, Well-Dispersed ZIF-Derived Co,N-Co-doped Carbon Nanoframes through Mesoporous-Silica-Protected Calcination as Efficient Oxygen Reduction Electrocatalysts, Advanced Materials, 28 (2016) 1668-1674.

[69] F. Hillman, J.M. Zimmerman, S.-M. Paek, M.R.A. Hamid, W.T. Lim, H.-K. Jeong, Rapid microwave-assisted synthesis of hybrid zeolitic-imidazolate frameworks with mixed metals and mixed linkers, Journal of Materials Chemistry A, 5 (2017) 6090-6099.

[70] H. Wang, C.Y. Ralston, D.S. Patil, R.M. Jones, W. Gu, M. Verhagen, M. Adams, P. Ge, C. Riordan, C.A. Marganian, P. Mascharak, J. Kovacs, C.G. Miller, T.J. Collins, S. Brooker, P.D. Croucher, K. Wang, E.I. Stiefel, S.P. Cramer, Nickel L-Edge Soft X-ray Spectroscopy of Nickel-Iron Hydrogenases and Model CompoundsEvidence for High-Spin Nickel(II) in the Active Enzyme, Journal of the American Chemical Society, 122 (2000) 10544-10552.

[71] A. Sharma, M. Varshney, H.J. Shin, B.-H. Lee, K.H. Chae, S.O. Won, Effect of Cu insertion on structural, local electronic/atomic structure and photocatalyst properties of $\mathrm{TiO} 2$, $\mathrm{ZnO}$ and $\mathrm{Ni}(\mathrm{OH}) 2$ nanostructures: XANES-EXAFS study, Materials Chemistry and Physics, 191 (2017) 129-144.

[72] G. Frank de, V. György, G. Pieter, The 1s x-ray absorption pre-edge structures in transition metal oxides, Journal of Physics: Condensed Matter, 21 (2009) 104207.

[73] N.L. Torad, M. Hu, S. Ishihara, H. Sukegawa, A.A. Belik, M. Imura, K. Ariga, Y. Sakka, Y. Yamauchi, Direct Synthesis of MOF-Derived Nanoporous Carbon with Magnetic Co Nanoparticles toward Efficient Water Treatment, Small, 10 (2014) 2096-2107.

[74] B. Liu, H. Shioyama, T. Akita, Q. Xu, Metal-Organic Framework as a Template for Porous Carbon Synthesis, Journal of the American Chemical Society, 130 (2008) 5390-5391. 
[75] Y.J. Sa, D.-J. Seo, J. Woo, J.T. Lim, J.Y. Cheon, S.Y. Yang, J.M. Lee, D. Kang, T.J. Shin, H.S. Shin, H.Y. Jeong, C.S. Kim, M.G. Kim, T.-Y. Kim, S.H. Joo, A General Approach to Preferential Formation of Active Fe-Nx Sites in Fe-N/C Electrocatalysts for Efficient Oxygen Reduction Reaction, Journal of the American Chemical Society, 138 (2016) 1504615056 . 